\title{
On a Fixed Point Theorem with Application to Integral Equations
}

\author{
Muhammad Nazam and Muhammad Arshad \\ Department of Mathematics, International Islamic University Islamabad, Islamabad 44000, Pakistan \\ Correspondence should be addressed to Muhammad Nazam; nazim.phdma47@iiu.edu.pk \\ Received 13 April 2016; Accepted 28 April 2016 \\ Academic Editor: Shamsul Qamar
}

Copyright ( 2016 M. Nazam and M. Arshad. This is an open access article distributed under the Creative Commons Attribution License, which permits unrestricted use, distribution, and reproduction in any medium, provided the original work is properly cited.

We introduce the notion of dualistic Geraghty's type contractions. We prove some fixed point theorems for ordered mappings satisfying the abovementioned contractions. We discuss an application of our fixed point results to show the existence of solution of integral equations.

\section{Introduction and Preliminaries}

In [1], Matthews introduced the concept of partial metric space as a suitable mathematical tool for program verification and proved an analogue of Banach fixed point theorem in complete partial metric spaces. O'Neill [2] introduced the concept of dualistic partial metric, which is more general than partial metric, and established a robust relationship between dualistic partial metric and quasi metric. In [3], Valero presented a Banach fixed point theorem on complete dualistic partial metric spaces. Valero also showed that the contractive condition in Banach fixed point theorem in complete dualistic partial metric spaces cannot be replaced by the contractive condition of Banach fixed point theorem for complete partial metric spaces. Later, Valero [3] generalized the main theorem of [4] using nonlinear contractive condition instead of Banach contractive condition.

For the sake of completeness, we recall Geraghty's Theorem. For this purpose, we first recall the class $S$ of all functions $\beta:[0, \infty) \rightarrow[0,1)$ which satisfies the condition

$$
\lim _{n \rightarrow \infty} \beta\left(t_{n}\right)=1 \text { implies } \lim _{n \rightarrow \infty} t_{n}=0 .
$$

In [5], Geraghty presented a new class of mappings $T$ : $M \rightarrow M$, called Geraghty contraction, which satisfies the following condition:

$$
d(T(j), T(k)) \leq \beta(d(j, k)) d(j, k),
$$

for all $j, k \in M$, where $\beta \in S$.
For this new family of mappings, Geraghty in [5] proved a fixed point theorem, stated below.

Theorem 1 (see [5]). Let $(M, d)$ be a complete metric space and let $T: M \rightarrow M$ be a mapping. Assume that there exists $\beta \in S$ such that, for all $j, k \in M$,

$$
d(T(j), T(k)) \leq \beta(d(j, k)) d(j, k) .
$$

Then, $T$ has a unique fixed point $v \in M$ and, for any choice of the initial point $j_{0} \in M$, the sequence $\left\{j_{n}\right\}$ defined by $j_{n}=$ $T\left(j_{n-1}\right)$ for each $n \geq 1$ converges to the point $v$.

Following [5], Amini-Harandi and Emami generalized Theorem 1 in context of ordered metric spaces (see [6]).

Theorem 2 (see [6]). Let $(M, \preceq)$ be an ordered set and suppose that there exists a metric $d$ in $M$ such that $(M, d)$ is a complete metric space. Let $T: M \rightarrow M$ be an increasing mapping such that there exists $j_{0} \in M$ with $j_{0} \preceq T\left(j_{0}\right)$. Suppose that there exists $\beta \in S$ such that

$$
\begin{array}{r}
d(T(j), T(k)) \leq \beta(d(j, k)) d(j, k) \\
\forall j, k \in M \text { with } j \geq k .
\end{array}
$$

Assume that either $T$ is continuous or $M$ is such that if an increasing sequence $\left\{j_{n}\right\}$ converges to $u$, then $j_{n} \preceq u$ for each $n \geq 1$. 
Besides, if for all $j, k \in M$ there exists $z \in M$ which is comparable to $j$ and $k$, then $T$ has a unique fixed point in $M$.

In [7], La Rosa and Vetro have extended the notion of Geraghty contraction mappings to the context of partial metric spaces. Besides, they have yielded partial metric version of Theorem 1, stated below.

Theorem 3 (see [7]). Let $(M, p)$ be a complete partial metric space and let $T: M \rightarrow M$ be a Geraghty contraction mapping. Then, $T$ has a unique fixed point $j \in M$ and the Picard iterative sequence $\left\{T^{n}\left(j_{0}\right)\right\}_{n \in \mathbb{N}}$ converges to $v$ with respect to $\tau\left(p^{s}\right)$, for any $j_{0} \in M$. Moreover, $p(v, v)=0$.

In this paper, we will present Theorems 1, 2, and 3 in dualistic partial metric spaces. We will show that our results generalize Theorems 1, 2, and 3 in many ways. In the section, we will apply our fixed point theorem to show the existence of solution of particular class of integral equations:

$$
j(w)=g(w)+\int_{0}^{1} G_{n}(w, s, j(s)) d s \quad \forall w \in[0,1] .
$$

We need some mathematical basics of dualistic partial metric space and results to make this paper self-sufficient.

Throughout, in this paper, the letters $\mathbb{R}^{+}, \mathbb{R}$, and $\mathbb{N}$ will represent the set of nonnegative real numbers, the set of real numbers, and the set of natural numbers, respectively.

According to O'Neill, a dualistic partial metric can be defined as follows.

Definition 4 (see [2]). Let $M$ be a nonempty set. If a function $D: M \times M \rightarrow \mathbb{R}$ satisfies, for all $j, k, l \in M$, the following properties:

$$
\begin{aligned}
& \left(D_{1}\right) j=k \Leftrightarrow D(j, j)=D(k, k)=D(j, k), \\
& \left(D_{2}\right) D(j, j) \leq D(j, k), \\
& \left(D_{3}\right) D(j, k)=D(k, j), \\
& \left(D_{4}\right) D(j, l)+D(k, k) \leq D(j, k)+D(k, l),
\end{aligned}
$$

then $D$ is called dualistic partial metric and the pair $(M, D)$ is known as dualistic partial metric space.

If $(M, D)$ is a dualistic partial metric space, then $d_{D}: M \times$ $M \rightarrow \mathbb{R}^{+}$defined by

$$
d_{D}(j, k)=D(j, k)-D(j, j)
$$

is called a dualistic quasi metric on $M$ such that $\tau(D)=$ $\tau\left(d_{D}\right) \forall j, k \in M$. Moreover, if $d_{D}$ is a dualistic quasi metric on $M$, then $d_{D}^{s}(j, k)=\max \left\{d_{D}(j, k), d_{D}(k, j)\right\}$ is a metric on $M$.

Remark 5. It is obvious that every partial metric is a dualistic partial metric but the converse is not true. To support this comment, define $D_{\vee}: \mathbb{R} \times \mathbb{R} \rightarrow \mathbb{R}$ by

$$
D_{\vee}(x, y)=x \vee y=\sup \{x, y\} \quad \forall x, y \in \mathbb{R} \text {. }
$$

It is easy to check that $D_{\vee}$ is a dualistic partial metric. Note that $D_{\vee}$ is not a partial metric, because $D_{\vee}(-1,-2)=-1 \notin$ $\mathbb{R}^{+}$. However, the restriction of $D_{\vee}$ to $\mathbb{R}^{+},\left.D_{\vee}\right|_{\mathbb{R}^{+}}$, is a partial metric.

Example 6. If $(M, d)$ is a metric space and $c \in \mathbb{R}$ is an arbitrary constant, then

$$
D(j, k)=d(j, k)+c
$$

defines a dualistic partial metric on $M$.

Following [2], each dualistic partial metric $D$ on $M$ generates a $T_{0}$ topology $\tau(D)$ on $M$. The topology $\tau(D)$ consists of open balls of the form $\left\{B_{D}(j, \varepsilon): j \in M, \varepsilon>0\right\}$, where $B_{D}(j, \varepsilon)=\{k \in M: D(j, k)<\varepsilon+D(j, j)\}$.

Definition 7 (see [2]). Let $(M, D)$ be a dualistic partial metric space:

(1) A sequence $\left\{j_{n}\right\}_{n \in \mathbb{N}}$ in $(M, D)$ converges to a point $j \in$ $M$ if and only if $D(j, j)=\lim _{n \rightarrow \infty} D\left(j, j_{n}\right)$.

(2) A sequence $\left\{j_{n}\right\}_{n \in \mathbb{N}}$ in $(M, D)$ is called a Cauchy sequence if $\lim _{n, m \rightarrow \infty} D\left(j_{n}, j_{m}\right)$ exists and is finite.

(3) A dualistic partial metric space $(M, D)$ is said to be complete if every Cauchy sequence $\left\{j_{n}\right\}_{n \in \mathbb{N}}$ in $M$ converges, with respect to $\tau(D)$, to a point $j \in M$ such that $D(j, j)=\lim _{n, m \rightarrow \infty} D\left(j_{n}, j_{m}\right)$.

The following lemma will be helpful in the sequel.

Lemma 8 (see $[2,3])$.

(1) A dualistic partial metric $(M, D)$ is complete if and only if the metric space $\left(M, d_{D}^{s}\right)$ is complete.

(2) A sequence $\left\{j_{n}\right\}_{n \in \mathbb{N}}$ in $M$ converges to a point $j \in M$, with respect to $\tau\left(d_{D}^{s}\right)$ if and only if $\lim _{n \rightarrow \infty} D\left(j, j_{n}\right)=$ $D(j, j)=\lim _{n \rightarrow \infty} D\left(j_{n}, j_{m}\right)$.

(3) If $\lim _{n \rightarrow \infty} j_{n}=v$ such that $D(v, v)=0$, then $\lim _{n \rightarrow \infty} D\left(j_{n}, k\right)=D(v, k)$ for every $k \in M$.

Oltra and Valero, in [4], established a Banach fixed point theorem, stated as follows.

Theorem 9 (see [4]). Let $(M, D)$ be a complete dualistic partial metric space and let $T: M \rightarrow M$ be a mapping such that there exists $\alpha \in[0,1[$ satisfying

$$
|D(T(j), T(k))| \leq \alpha|D(j, k)|,
$$

for all $j, k \in M$. Then, $T$ has a unique fixed point $v \in$ M. Moreover, $D(v, v)=0$ and the Picard iterative sequence $\left\{T^{n}\left(j_{0}\right)\right\}_{n \in \mathbb{N}}$ converges to $v$ with respect to $\tau\left(d_{D}^{s}\right)$, for any $j_{0} \in$ $M$.

\section{The Results}

In this section, we will prove the existence of fixed points of dualistic contractions in ordered dualistic partial metric spaces. To this end, we need to define the followings notions. 
Definition 10 . Let $M$ be a nonempty set. Then, $(M, \preceq, D)$ is said to be an ordered dualistic partial metric space if

(i) $(M, \preceq)$ is a partially ordered set;

(ii) $(M, D)$ is a dualistic partial metric space.

Definition 11. Let $(M, \preceq)$ be a partially ordered set and suppose that $(M, D)$ is a complete dualistic partial metric space; a mapping $T: M \rightarrow M$ is called dualistic Geraghty's type contraction provided there exists $\beta \in S$ such that

$$
|D(T(j), T(k))| \leq \beta(|D(j, k)|)|D(j, k)|,
$$

for all comparable $j, k \in M$.

Now we present our main result.

Theorem 12. Let $(M, \preceq)$ be a partially ordered set and suppose that $(M, D)$ is a complete dualistic partial metric space and let $T: M \rightarrow M$ be a mapping such that

(1) $T$ is a dominated mapping;

(2) $T$ is a dualistic Geraghty's type contraction;

(3) either $T$ is continuous or if $\left\{j_{n}\right\}$ is a nonincreasing sequence in $M$ such that $\left\{j_{n}\right\} \rightarrow v$, then $j_{n} \geq v \forall n$.

Then, $T$ has a fixed point $v \in M$ and the Picard iterative sequence $\left\{T^{n}(j)\right\}_{n \in \mathbb{N}}$ converges to $v$ with respect to $\tau\left(d_{D}^{s}\right)$, for every $j \in M$. Moreover, $D(v, v)=0$.

Proof. Let $j_{0} \in M$ be an initial element and $j_{n}=T\left(j_{n-1}\right)$ for all $n \geq 1$; if there exists a positive integer $r$ such that $j_{r+1}=j_{r}$, then $j_{r}=T\left(j_{r}\right)$, so we are done. Suppose that $j_{n} \neq j_{n+1} \forall n \in \mathbb{N}$; then, since $T$ is dominated mapping, therefore $j_{0} \geq T\left(j_{0}\right)=j_{1}$; that is $j_{0} \succeq j_{1}$ and $j_{1} \geq T\left(j_{1}\right)$ imply $j_{1} \geq j_{2}$ and $j_{2} \geq T\left(j_{2}\right)$ implies $j_{2} \geq j_{3}$; continuing in a similar way, we get

$$
j_{0} \geq j_{1} \geq j_{2} \geq j_{3} \geq \cdots \geq j_{n} \geq j_{n+1} \geq j_{n+2} \geq \cdots .
$$

Since $j_{n} \geq j_{n+1}$, from contractive condition (10) we have

$$
\begin{aligned}
\left|D\left(j_{n+1}, j_{n+2}\right)\right| & =\left|D\left(T\left(j_{n}\right), T\left(j_{n+1}\right)\right)\right| \\
& \leq \beta\left(\left|D\left(j_{n}, j_{n+1}\right)\right|\right)\left|D\left(j_{n}, j_{n+1}\right)\right| \\
& \leq\left|D\left(j_{n}, j_{n+1}\right)\right| \quad \forall n \geq 1 .
\end{aligned}
$$

This implies that $\left\{\left|D\left(j_{n}, j_{n+1}\right)\right|\right\}_{n=1}^{\infty}$ is a monotone and bounded above sequence; it is convergent and converges to a point $\alpha$; that is,

$$
\lim _{n \rightarrow \infty}\left|D\left(j_{n}, j_{n+1}\right)\right|=\alpha \geq 0 .
$$

If $\alpha=0$, then we are done but if $\alpha>0$, then from (10) we have

$$
\left|D\left(j_{n+1}, j_{n+2}\right)\right| \leq \beta\left(\left|D\left(j_{n}, j_{n+1}\right)\right|\right)\left|D\left(j_{n}, j_{n+1}\right)\right| .
$$

This implies that

$$
\left|\frac{D\left(j_{n+1}, j_{n+2}\right)}{D\left(j_{n}, j_{n+1}\right)}\right| \leq \beta\left(\left|D\left(j_{n}, j_{n+1}\right)\right|\right) .
$$

Taking limit, we have

$$
\lim _{n \rightarrow \infty} \beta\left(\left|D\left(j_{n}, j_{n+1}\right)\right|\right)=1 \text {. }
$$

Since $\beta \in S, \lim _{n \rightarrow \infty}\left|D\left(j_{n}, j_{n+1}\right)\right|=0$ which entails $\alpha=0$.

Hence,

$$
\lim _{n \rightarrow \infty} D\left(j_{n}, j_{n+1}\right)=0 \text {. }
$$

Similarly, we can prove that

$$
\lim _{n \rightarrow \infty} D\left(j_{n}, j_{n}\right)=0
$$

Now since

$$
d_{D}\left(j_{n}, j_{n+1}\right)=D\left(j_{n}, j_{n+1}\right)-D\left(j_{n}, j_{n}\right),
$$

we deduce that

$$
\lim _{n \rightarrow \infty} d_{D}\left(j_{n}, j_{n+1}\right)=0 \quad \forall n \geq 1 .
$$

Now, we show that sequence $\left\{j_{n}\right\}$ is a Cauchy sequence $\left(M, d_{D}^{s}\right)$. Suppose on the contrary that $\left\{j_{n}\right\}$ is not a Cauchy sequence. Then, given $\varepsilon>0$, we will construct a pair of subsequences $\left\{j_{m_{r}}\right\}$ and $\left\{j_{n_{r}}\right\}$ violating the following condition for least integer $n_{r}$ such that $m_{r}>n_{r}>r$, where $r \in \mathbb{N}$ :

$$
d_{D}\left(j_{m_{r}}, j_{n_{r}}\right) \geq \varepsilon
$$

In addition, upon choosing the smallest possible $m_{r}$, we may assume that

$$
d_{D}\left(j_{m_{r}}, j_{n_{r-1}}\right)<\varepsilon
$$

By the triangle inequality, we have

$$
\begin{aligned}
\varepsilon & \leq d_{D}\left(j_{m_{r}}, j_{n_{r}}\right) \leq d_{D}\left(j_{m_{r}}, j_{n_{r-1}}\right)+d_{D}\left(j_{n_{r-1}}, j_{n_{r}}\right) \\
& <\varepsilon+d_{D}\left(j_{n_{r-1}}, j_{n_{r}}\right) .
\end{aligned}
$$

That is,

$$
\varepsilon<\varepsilon+d_{D}\left(j_{n_{r-1}}, j_{n_{r}}\right)
$$

for all $r \in \mathbb{N}$. In the view of (24) and (17), we have

$$
\lim _{r \rightarrow \infty} d_{D}\left(j_{m_{r}}, j_{n_{r}}\right)=\varepsilon
$$

Again using triangle inequality, we have

$$
\begin{aligned}
d_{D}\left(j_{m_{r}}, j_{n_{r}}\right) \leq & d_{D}\left(j_{m_{r}}, j_{m_{r+1}}\right)+d_{D}\left(j_{m_{r+1}}, j_{n_{r+1}}\right) \\
& +d_{D}\left(j_{n_{r+1}}, j_{n_{r}}\right), \\
d_{D}\left(j_{m_{r+1}}, j_{n_{r+1}}\right) \leq & d_{D}\left(j_{m_{r+1}}, j_{m_{r}}\right)+d_{D}\left(j_{m_{r}}, j_{n_{r}}\right) \\
& +d_{D}\left(j_{n_{r}}, j_{n_{r+1}}\right) .
\end{aligned}
$$

Taking limit as $r \rightarrow+\infty$ and using (17) and (25), we obtain

$$
\lim _{r \rightarrow+\infty} d_{D}\left(j_{m_{r+1}}, j_{n_{r+1}}\right)=\varepsilon
$$


Now from contractive condition (10), we have

$$
\begin{aligned}
\left|D\left(j_{n_{r+1}}, j_{m_{r+2}}\right)\right| & =\left|D\left(T\left(j_{n_{r}}\right), T\left(j_{m_{r+1}}\right)\right)\right| \\
& \leq \beta\left(\left|D\left(j_{n_{r}}, j_{m_{r+1}}\right)\right|\right)\left|D\left(j_{n_{r}}, j_{m_{r+1}}\right)\right| .
\end{aligned}
$$

We conclude that

$$
\left|\frac{D\left(j_{n_{r+1}}, j_{m_{r+2}}\right)}{D\left(j_{n_{r}}, j_{m_{r+1}}\right)}\right| \leq \beta\left(\left|D\left(j_{n_{r}}, j_{m_{r+1}}\right)\right|\right) .
$$

By using (17), letting $r \rightarrow+\infty$ in the above inequality, we obtain

$$
\lim _{r \rightarrow \infty} \beta\left(\left|D\left(j_{n_{r}}, j_{m_{r+1}}\right)\right|\right)=1 .
$$

Since $\beta \in S, \lim _{r \rightarrow \infty}\left|D\left(j_{n_{r}}, j_{m_{r+1}}\right)\right|=0$ and hence $\lim _{r \rightarrow \infty} d_{D}\left(j_{n_{r}}, j_{m_{r+1}}\right)=0<\varepsilon$ which contradicts our assumption (21). Arguing like above, we can have $\lim _{r \rightarrow \infty} d_{D}\left(j_{m_{r}}\right.$, $\left.j_{n_{r+1}}\right)=0<\varepsilon$. Hence, $\left\{j_{n}\right\}$ is a Cauchy sequence in $\left(M, d_{D}^{s}\right)$; that is, $\lim _{n, m \rightarrow \infty} d_{D}^{s}\left(j_{n}, j_{m}\right)=0$. Since $\left(M, d_{D}^{s}\right)$ is a complete metric space, $\left\{j_{n}\right\}$ converges to a point $v$ in $M$; that is, $\lim _{n \rightarrow \infty} d_{D}^{s}\left(j_{n}, v\right)=0$; then, from Lemma 8 , we get

$$
\lim _{n \rightarrow \infty} D\left(v, j_{n}\right)=D(v, v)=\lim _{n, m \rightarrow \infty} D\left(j_{n}, j_{m}\right)=0 .
$$

We are left to prove that $v$ is a fixed point of $T$. For this purpose, we have to deal with two cases.

Case 1 . If $T$ is continuous, then

$$
\begin{aligned}
v & =\lim _{n \rightarrow \infty} j_{n}=\lim _{n \rightarrow \infty} T^{n}\left(j_{0}\right)=\lim _{n \rightarrow \infty} T^{n+1}\left(j_{0}\right) \\
& =T\left(\lim _{n \rightarrow \infty} T^{n}\left(j_{0}\right)\right)=T(v) .
\end{aligned}
$$

Hence, $v=T(v)$; that is, $v$ is a fixed point of $T$.

Case 2. If $\left\{j_{n}\right\}$ is a nonincreasing sequence in $M$ such that $\left\{j_{n}\right\} \rightarrow v$, then $j_{n} \geq v \forall n$.

Using contractive condition (10) and (31), we get

$$
\begin{aligned}
& \left|D\left(j_{n+1}, T(v)\right)\right|=\left|D\left(T\left(j_{n}\right), T(v)\right)\right| \\
& \quad \leq \beta\left(\left|D\left(j_{n}, v\right)\right|\right)\left|D\left(j_{n}, v\right)\right|, \\
& \lim _{n \rightarrow \infty}\left|D\left(j_{n+1}, T(v)\right)\right| \leq \lim _{n \rightarrow \infty} \beta\left(D\left(j_{n}, v\right)\right) D\left(j_{n}, v\right) \\
& \text { which implies }|D(v, T(v))| \leq 0 .
\end{aligned}
$$

This shows that $D(v, T(v))=0$. So from $\left(D_{1}\right)$ and $\left(D_{2}\right)$, we deduce that $v=T(v)$ and hence $v$ is a fixed point of $T$.

Note that in the above result fixed point may not be unique; in order to prove uniqueness of the fixed point, we need some more conditions and for this purpose we have following theorem.

Theorem 13. Let $(M, \preceq, D)$ be an ordered complete dualistic partial metric space. Let $T: M \rightarrow M$ be a mapping satisfying all the conditions of Theorem 12. Besides, if for each $j, k \in M$ there exists $z \in M$ which is comparable to $j$ and $k$, then $T$ has a unique fixed point.
Proof. Following the proof of Theorem 12, we know that $v$ is a fixed point of $T$. We are left to prove the uniqueness of the fixed point $v$. Let $v_{1}$ be another fixed point of $T$; then, $T\left(v_{1}\right)=v_{1}$. Two cases arise; first if $v$ and $v_{1}$ are comparable, then from (10) it follows that $v=v_{1}$. Secondly, if $v$ and $v_{1}$ are not comparable, then there exists $z \in M$ which is comparable to both $v$ and $v_{1}$; that is, $v \geq z$ and $v_{1} \geq z$. Since $T$ is dominated mapping, we deduce that $v \geq T^{n}(z)$ and $v_{1} \geq$ $T^{n}(z)$. Moreover, consider $\left|D\left(v, T^{n}(z)\right)\right|=\left|D\left(T^{n}(v), T^{n}(z)\right)\right|$ and by contractive condition (10), we obtain

$$
\begin{aligned}
& \left|D\left(T^{n}(v), T^{n}(z)\right)\right| \leq \beta\left(\left|D\left(T^{n-1}(v), T^{n-1}(z)\right)\right|\right) \\
& \cdot\left|D\left(T^{n-1}(v), T^{n-1}(z)\right)\right| .
\end{aligned}
$$

This implies

$$
\left|D\left(v, T^{n}(z)\right)\right| \leq\left|D\left(v, T^{n-1}(z)\right)\right| .
$$

It shows that $\left\{\left|D\left(v, T^{n}(z)\right)\right|\right\}_{n=1}^{\infty}$ is a nonnegative and decreasing sequence, so for $\lambda \geq 0$ we get

$$
\lim _{n \rightarrow \infty}\left|D\left(v, T^{n}(z)\right)\right|=\lambda \text {. }
$$

We claim that $\lambda=0$. Suppose on the contrary that $\lambda>0$.

By passing to subsequences, if necessary, we may assume that

$$
\lim _{n \rightarrow \infty} \beta\left(\left|D\left(v, T^{n}(z)\right)\right|\right)=\gamma .
$$

Then, by (34) we have $\lambda \leq \gamma \lambda \Rightarrow \gamma=1$. Since $\beta \in S$, we have

$$
\lim _{n \rightarrow \infty}\left|D\left(v, T^{n}(z)\right)\right|=0 \text {. }
$$

Hence,

$$
\lim _{n \rightarrow \infty} D\left(v, T^{n}(z)\right)=0
$$

Similarly, we can prove that

$$
\lim _{n \rightarrow \infty} D\left(v_{1}, T^{n}(z)\right)=0 .
$$

Finally, by $D_{4}$ we have

$$
\begin{aligned}
D\left(v, v_{1}\right) \leq & D\left(v, T^{n}(z)\right)+D\left(T^{n}(z), v_{1}\right) \\
& -D\left(T^{n}(z), T^{n}(z)\right) \\
\leq & D\left(v_{1}, T^{n}(z)\right)+D\left(T^{n}(z), v\right) \\
& -D\left(T^{n}(z), v\right)-D\left(v, T^{n}(z)\right)+D(v, v) .
\end{aligned}
$$

Taking limit, we get $D\left(v, v_{1}\right) \leq 0$, since $d_{D}\left(v, v_{1}\right)=D\left(v, v_{1}\right)-$ $D\left(v_{1}, v_{1}\right)$, which implies $D\left(v, v_{1}\right) \geq 0$. Hence, $D\left(v, v_{1}\right)=0$. From $D_{1}$ and $D_{2}$, we deduce that $v=v_{1}$ which proves the uniqueness of $v$.

For monotone mappings, we present following result.

Theorem 14. Let $(M, \preceq)$ be a partially ordered set and suppose that $(M, D)$ is a complete dualistic partial metric space and let $T: M \rightarrow M$ be a mapping such that

(1) $T$ is an increasing map with $j_{0} \preceq T\left(j_{0}\right)$ for some $j_{0} \in$ $M$; 
(2) $T$ is a dualistic Geraghty's type contraction;

(3) either $T$ is continuous or $M$ is such that if an increasing sequence $\left\{j_{n}\right\} \rightarrow u \in M$ then $j_{n} \preceq u$.

Besides, if for each $j, k \in M$ there exists $z \in M$ which is comparable to $j$ and $k$, then $T$ has a unique fixed point $v \in M$ and the Picard iterative sequence $\left\{T^{n}\left(j_{0}\right)\right\}_{n \in \mathbb{N}}$ converges to $v$ with respect to $\tau\left(d_{D}^{s}\right)$, for any $j_{0} \in M$. Moreover, $D(v, v)=0$.

Proof. We begin by defining a Picard iterative sequence in $M$ by $j_{n}=T\left(j_{n-1}\right)$ for all $n \in \mathbb{N}$. Given $j_{0} \preceq T\left(j_{0}\right)=j_{1}$, then $j_{0} \preceq j_{1}$. Since $T$ is increasing, $j_{0} \preceq j_{1}$ implies $T\left(j_{0}\right) \preceq T\left(j_{1}\right)$; that is, $j_{1} \preceq j_{2}$; this in turn gives $T\left(j_{1}\right) \preceq T\left(j_{2}\right)$ which implies $j_{2} \preceq j_{3}$. Continuing in a similar way we get

$$
j_{0} \preceq j_{1} \preceq j_{2} \preceq j_{3} \preceq \cdots \preceq j_{n} \preceq j_{n+1} \cdots .
$$

Since $j_{n} \preceq j_{n+1}$ for each $n \in \mathbb{N}$, from contractive condition (10) we have

$$
\begin{aligned}
\left|D\left(j_{n+1}, j_{n+2}\right)\right| & =\left|D\left(T\left(j_{n}\right), T\left(j_{n+1}\right)\right)\right| \\
& \leq \beta\left(\left|D\left(j_{n}, j_{n+1}\right)\right|\right)\left|D\left(j_{n}, j_{n+1}\right)\right| \\
& \leq\left|D\left(j_{n}, j_{n+1}\right)\right| \quad \forall n \geq 1 .
\end{aligned}
$$

This implies that $\left\{\left|D\left(j_{n}, j_{n+1}\right)\right|\right\}_{n=1}^{\infty}$ is a monotone and bounded below sequence; it is convergent and converges to a point $\alpha$; that is,

$$
\lim _{n \rightarrow \infty}\left|D\left(j_{n}, j_{n+1}\right)\right|=\alpha \geq 0 .
$$

If $\alpha=0$, then we are done but if $\alpha>0$, then from (10) we have

$$
\left|D\left(j_{n+1}, j_{n+2}\right)\right| \leq \beta\left(\left|D\left(j_{n}, j_{n+1}\right)\right|\right)\left|D\left(j_{n}, j_{n+1}\right)\right| .
$$

This implies that

$$
\left|\frac{D\left(j_{n+1}, j_{n+2}\right)}{D\left(j_{n}, j_{n+1}\right)}\right| \leq \beta\left(\left|D\left(j_{n}, j_{n+1}\right)\right|\right) .
$$

Taking limit, we have

$$
\lim _{n \rightarrow \infty} \beta\left(\left|D\left(j_{n}, j_{n+1}\right)\right|\right)=1 \text {. }
$$

Since $\beta \in S, \lim _{n \rightarrow \infty}\left|D\left(j_{n}, j_{n+1}\right)\right|=0$ which implies $\alpha=0$.

Hence,

$$
\lim _{n \rightarrow \infty} D\left(j_{n}, j_{n+1}\right)=0 \text {. }
$$

Similarly, we can prove that

$$
\lim _{n \rightarrow \infty} D\left(j_{n}, j_{n}\right)=0 .
$$

And the desired conclusion follows arguing like in the proofs of Theorems 12 and 13. 14.

Clearly, Theorem 3 is a particular case of Theorems 12 and

A natural question that can be raised is whether the contractive condition in the statements of Theorems 12 and 14 can be replaced by the contractive condition in Theorem 3 . The following easy example provides a negative answer to such a question.
Example 15. Consider the complete dualistic partial metric $\left(\mathbb{R}, D_{\vee}\right)$ and the mapping $T_{0}: \mathbb{R} \rightarrow \mathbb{R}$ defined by

$$
T_{0}(j)= \begin{cases}0 & \text { if } j \neq 0 \\ -1 & \text { if } j=0 .\end{cases}
$$

It is easy to check that the contractive condition in the statement of Theorem 3 holds:

$$
D_{\vee}\left(T_{0}(j), T_{0}(k)\right) \leq \beta\left(D_{\vee}(j, k)\right) D_{\vee}(j, k)
$$

for all $j, k \in \mathbb{R}$. However, $T_{0}$ does not have a fixed point. Observe that $T_{0}$ does not satisfy the contractive condition in the statements of Theorems 12 and 14. Indeed,

$$
\begin{aligned}
1 & =\left|D_{\vee}(-1,-1)\right|=\left|D_{\vee}\left(T_{0}(0), T_{0}(0)\right)\right| \\
& >\beta\left(\left|D_{\vee}(0,0)\right|\right)\left|D_{\vee}(0,0)\right|=0 .
\end{aligned}
$$

The analogues of Theorems 12 and 14 are given below without proofs as they can be obtained easily by following proofs of above theorems.

Theorem 16. Let $(M, \preceq)$ be a partially ordered set and suppose that $(M, D)$ is a complete dualistic partial metric space and let $T: M \rightarrow M$ be a mapping such that

(1) $T$ is a dominating map;

(2) $T$ is a dualistic Geraghty's type contraction;

(3) either $T$ is continuous or $M$ is such that if an increasing sequence $\left\{j_{n}\right\} \rightarrow u \in M$ then $j_{n} \preceq u$.

Besides, if for each $j, k \in M$ there exists $z \in M$ which is comparable to $j$ and $k$, then, $T$ has a unique fixed point $v \in M$ and the Picard iterative sequence $\left\{T^{n}\left(j_{0}\right)\right\}_{n \in \mathbb{N}}$ converges to $v$ with respect to $\tau\left(d_{D}^{s}\right)$, for any $j_{0} \in M$. Moreover, $D(v, v)=0$.

Theorem 17. Let $(M, \preceq)$ be a partially ordered set and suppose that $(M, D)$ is a complete dualistic partial metric space and let $T: M \rightarrow M$ be a mapping such that

(1) $T$ is a decreasing map with $T\left(x_{0}\right) \preceq x_{0}$;

(2) $T$ is a dualistic Geraghty's type contraction;

(3) either $T$ is continuous or $M$ is such that if a decreasing sequence $\left\{j_{n}\right\} \rightarrow u \in M$ then $j_{n} \geq u$.

Besides, if for each $j, k \in M$ there exists $z \in M$ which is comparable to $j$ and $k$, then $T$ has a unique fixed point $v \in M$ and the Picard iterative sequence $\left\{T^{n}\left(j_{0}\right)\right\}_{n \in \mathbb{N}}$ converges to $v$ with respect to $\tau\left(d_{D}^{s}\right)$, for any $j_{0} \in M$. Moreover, $D(v, v)=0$.

Observations. If we set $D(j, j)=0$ in Theorem 14 , we retrieve Theorem 2 as a particular case. If we set $D(j, k) \in \mathbb{R}^{+}$in Theorem 14, we retrieve Theorem 3 as a particular case.

\section{Application to Integral Equations}

In this section, we will show how Theorem 14 can be applied to prove the existence of solution of integral equation (53).

Let $\Omega$ represent the class of functions $\varphi:[0, \infty) \rightarrow[0, \infty)$ with the following properties:

(1) $\varphi$ is increasing; 
(2) for each $j>0, \varphi(j)<j$;

(3) $\int_{0}^{1} \varphi(j) d j \leq \varphi\left(\int_{0}^{1} j d j\right)$;

(4) $\beta(j)=\varphi(j) / j \in S$.

For example, $\varphi(j)=(1 / 5) j$ and $\varphi(j)=\tan (j)$ are elements of $\Omega$.

Let us consider the following integral equation:

$$
j(w)=g(w)+\int_{0}^{1} G(w, s, j(s)) d s \quad \forall w \in[0,1] .
$$

To show the existence of solution of integral equation (53), we need following lemma.

Lemma 18. Let $\mathbb{B}=\bar{B}(0, \rho)=\left\{j: j \in L^{2}([0,1], \mathbb{R}) ;\|j\| \leq \rho\right\}$. Assume the following hypotheses are satisfied:

(1) $g \in L^{2}([0,1], \mathbb{R})$;

(2) $G:[0,1] \times[0,1] \times L^{2}([0,1], \mathbb{R}) \rightarrow \mathbb{R}$;

(3) $\left|G_{n}(w, s, j)\right| \leq f(w, s)+v|j|$, where $f \in L^{2}([0,1] \times$ $[0,1])$ and $v<1 / 2$.

Then, operator $T$ defined by

$$
(T k)(w)=g(w)+\int_{0}^{1} \widetilde{G}(w)(k)(s) d s
$$

satisfies $T(\mathbb{B}) \subset \mathbb{B}$.

Proof. We begin by defining the operator $\widetilde{G}(w)(k)(s)=$ $G_{n}(w, s, k(s))$ :

$$
\begin{aligned}
& \|T j\|_{L^{2}([0,1], \mathbb{R})}^{2}=\int_{0}^{1}|T j(w)|^{2} d w \\
& =\int_{0}^{1}\left(\left|g(w)+\int_{0}^{1} \widetilde{G}(w)(j)(s) d s\right|^{2}\right) d w \\
& \leq 2 \int_{0}^{1}|g(w)|^{2} d w+2 \iint_{0}^{1}|\widetilde{G}(w)(j)(s)|^{2} d s d w \\
& \leq 2 \int_{0}^{1}|g(w)|^{2} d w \\
& \quad+2 \iint_{0}^{1}|f(w, s)+v| j(s) \|^{2} d s d w \\
& \leq 2 \int_{0}^{1}|g(w)|^{2} d w+4 \iint_{0}^{1}|f(w, s)|^{2} d s d w \\
& \quad+4 v^{2}\|j\|_{L^{2}([0,1], \mathbb{R})}^{1}|g(w)|^{2} d w+4 \iint_{0}^{1} f^{2}(w, s) d s d w \\
& \leq 2 \int_{0}^{1} \mid 4 v^{2} \rho^{2} .
\end{aligned}
$$

Since $v<1 / 2$, choose $\rho$ such that

$$
\begin{aligned}
& \frac{2}{1-4 v^{2}} \int_{0}^{1}|g(w)|^{2} d w+\frac{4}{1-4 v^{2}} \iint_{0}^{1} f^{2}(w, s) d s d w \\
& \quad \leq \rho^{2} .
\end{aligned}
$$

This implies that $T(j) \in \mathbb{B}$; hence, $T(\mathbb{B}) \subset \mathbb{B}$.

Now we are in position to state our result regarding application.

Theorem 19. Assume that the following hypotheses are satisfied:

(1) The conditions supposed in Lemma 18 hold.

(2) $G_{n}(w, s, j)-G_{n}(w, s, k) \leq \varphi(j-k), \forall$ comparable $j, k \in$ $M$ and for large $n$.

Then, integral equation (53) has a solution.

Proof. Let $M=L^{2}([0,1], \mathbb{R})$ and $D(j, k)=d(j, k)+c_{n} \forall j, k \in$ $M$, where $d(j, k)=\|j-k\|_{M}$ and $\left\{c_{n}\right\}$ is a sequence of real numbers satisfying $\left|c_{n}\right| \rightarrow 0$ for large $n$. Suppose that $T$ : $M \rightarrow M$ is a mapping defined by

$$
(T k)(w)=g(w)+\int_{0}^{1} \widetilde{G}(w)(k)(s) d s .
$$

We introduce a partial ordering on $M$, setting

$$
u_{1} \preceq u_{2} \Longleftrightarrow u_{1}(w) \leq u_{2}(w) \quad \forall w \in[0,1] .
$$

Then, $(M, \preceq, D)$ is a complete ordered dualistic partial metric space. Notice that $T$ is well-defined and (53) has a solution if and only if the operator $T$ has a fixed point. Precisely, we have to show that Theorem 14 is applicable to the operator $T$. Then, for all comparable $j, k \in M$, we write

$$
\begin{aligned}
\mid D( & T(j), T(k))\left.\right|^{2}=\left|d(T(j), T(k))+c_{n}\right|^{2} \\
\leq & |d(T(j), T(k))|^{2}+\left|c_{n}\right|^{2} \\
& +2|d(T(j), T(k))|\left|c_{n}\right| \\
\leq & \|T(j)-T(k)\|^{2}+\left|c_{n}\right|^{2}+2|d(T(j), T(k))|\left|c_{n}\right| \\
\leq & \int_{0}^{1}\left(\int_{0}^{1} \widetilde{G}(w)(j)(s)-\widetilde{G}(w)(k)(s) d s\right)^{2} d w \\
& +\left|c_{n}\right|^{2}+2|d(T j, T k)|\left|c_{n}\right| \\
\leq & \int_{0}^{1}\left(\int_{0}^{1} G_{n}(w, s, j)-G_{n}(w, s, k) d s\right)^{2} d w \\
& +\left|c_{n}\right|^{2}+2|d(T(j), T(k))|\left|c_{n}\right| \\
\leq & \int_{0}^{1}\left(\int_{0}^{1} \varphi(j(s)-k(s)) d s\right)^{2} d w \text { for large } n \\
\leq & \varphi^{2}\left(\int_{0}^{1}(j(s)-k(s))^{2} d s\right) .
\end{aligned}
$$


It follows that

$$
\begin{aligned}
|D(T(j), T(k))|^{2} & \leq[\varphi(|D(j, k)|)]^{2} \\
|D(T(j), T(k))| & \leq[\varphi(|D(j, k)|)] \\
& =\frac{\varphi(|D(j, k)|)}{|D(j, k)|}|D(j, k)| .
\end{aligned}
$$

This implies

$$
|D(T(j), T(k))| \leq \beta(|D(j, k)|)|D(j, k)|
$$

and hence $T$ satisfies all the conditions of Theorem 14, so it has a fixed point and hence (53) has a solution.

Remark 20. The significance of the above results lies in the fact that these results are true for all real numbers whereas such results proved in partial metric spaces are only true for positive real numbers.

\section{Competing Interests}

The authors declare that they have no competing interests.

\section{References}

[1] S. G. Matthews, "Partial metric topology", in Proceedings of the 11th Summer Conference on General Topology and Applications, vol. 728, pp. 183-197, The New York Academy of Sciences, Flushing, NY, USA, August 1995.

[2] S. J. O'Neill, "Partial metric, valuations and domain theory," Annals of the New York Academy of Science, vol. 806, pp. 304315, 1996.

[3] O. Valero, "On Banach fixed point theorems for partial metric spaces," Applied General Topology, vol. 6, no. 2, pp. 229-240, 2005.

[4] S. Oltra and O. Valero, "Banach's fixed point theorem for partial metric spaces," Rendiconti dell'Istituto di Matematica dell'Universita di Trieste, vol. 36, no. 1-2, pp. 17-26, 2004.

[5] M. A. Geraghty, "On contractive mappings," Proceedings of the American Mathematical Society, vol. 40, pp. 604-608, 1973.

[6] A. Amini-Harandi and H. Emami, "A fixed point theorem for contraction type maps in partially ordered metric spaces and application to ordinary differential equations," Nonlinear Analysis: Theory, Methods \& Applications, vol. 72, no. 5, pp. 2238-2242, 2010.

[7] V. La Rosa and P. Vetro, "Fixed points for Geraghty-contractions in partial metric spaces," Journal of Nonlinear Science and Its Applications, vol. 7, no. 1, pp. 1-10, 2014. 


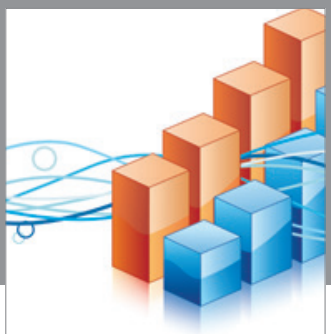

Advances in

Operations Research

vatem alat4

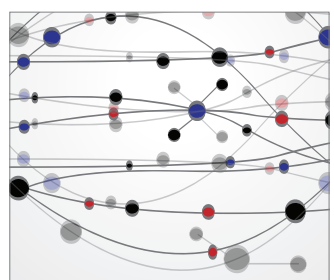

\section{The Scientific} World Journal
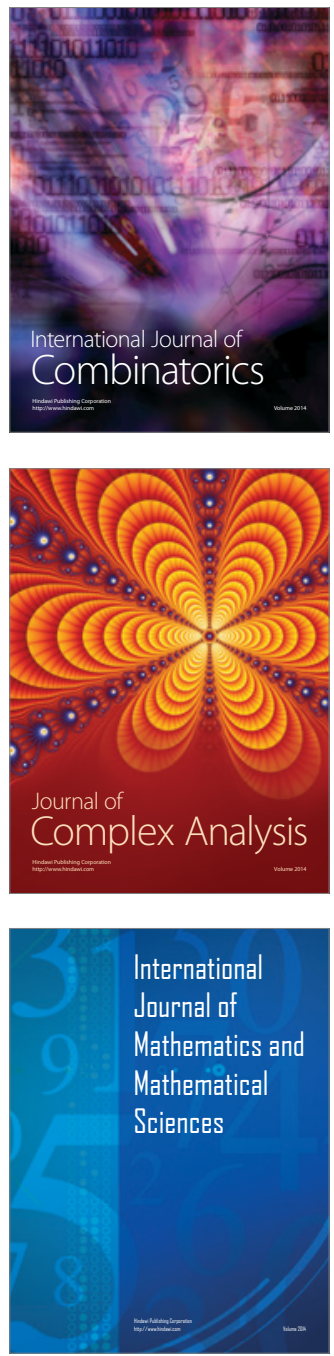
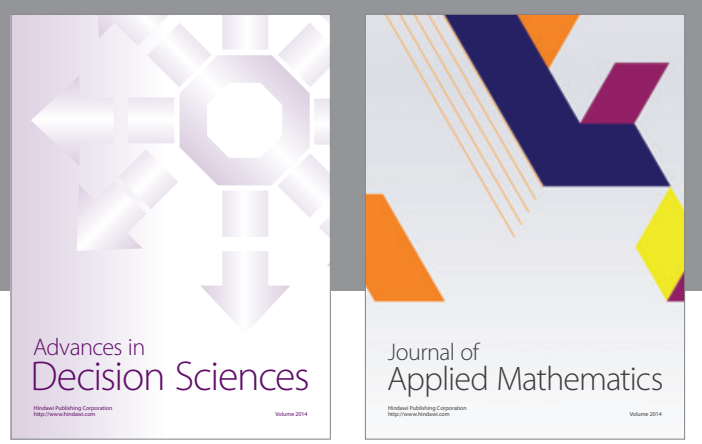

Algebra

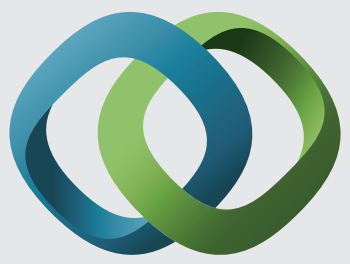

\section{Hindawi}

Submit your manuscripts at

http://www.hindawi.com
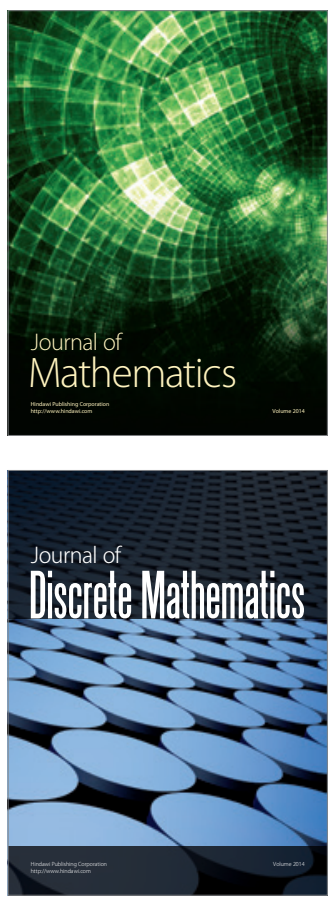

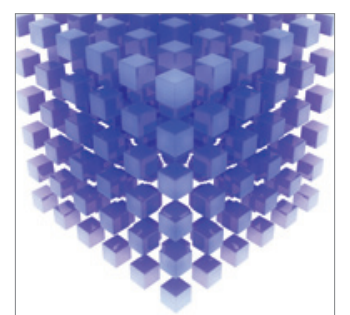

Mathematical Problems in Engineering
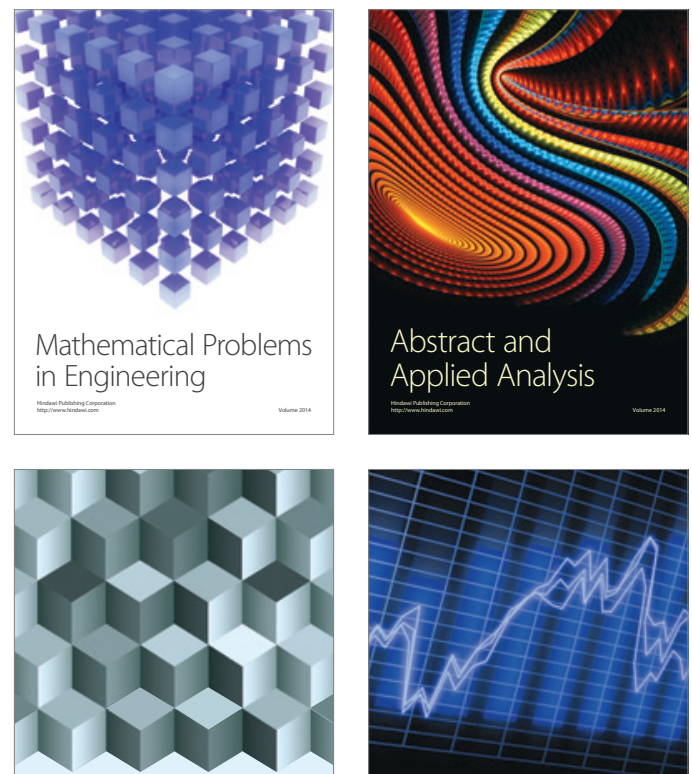

Journal of

Function Spaces

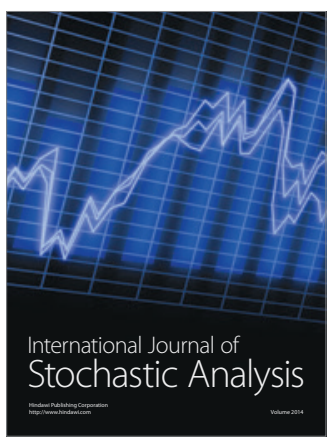

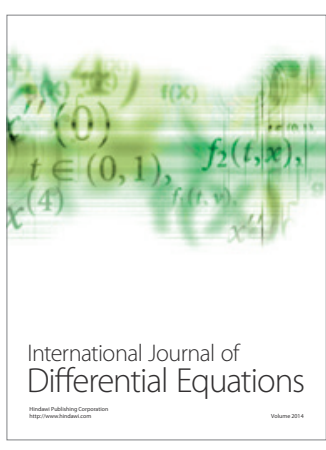
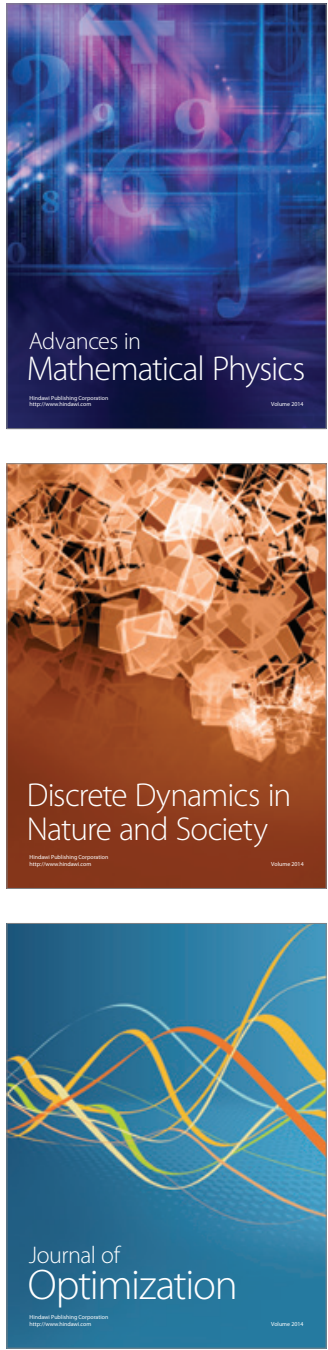